\title{
Emerging Therapies for Cutaneous Lupus Erythematosus
}

\author{
Robert Borucki, MD; Victoria P. Werth, MD
}

$\cup^{i}$ ystemic lupus erythematosus (SLE) is a chronic autoimmune inflammatory disease that can have devastating effects on many organs. Despite the considerable morbidity and mortality associated with SLE, treatment options have been largely unchanged since the 1950s. ${ }^{1}$ It was not until the last decade that a new biologic medication was approved, and several other promising treatments currently are being evaluated in clinical trials. Dermatologists are most likely to encounter cutaneous lupus erythematosus (CLE) with or without SLE, which can present with a variety of skin manifestations. Cutaneous lupus erythematosus can have devastating effects on quality of life and can be a visible sign of the internal activity and damage of SLE., Although many trials have been completed evaluating SLE treatments, few medications have been evaluated specifically for CLE despite the availability of validated measures of CLE skin activity. ${ }^{4}$ There is a recent shortage of antimalarial medications, the current first-line therapy for CLE, due to both an import alert in the United States on quinacrine placed in 2019 as well as the use of hydroxychloroquine and chloroquine in treating coronavirus disease 2019.5,6 Due to this shortage, the need for new and effective treatments is more critical than ever, as alternatives to first-line therapy frequently require immunosuppression. We review recent drug approvals for SLE and their efficacy in CLE. We also provide an update on new agents currently being studied to treat this disease.

\section{Belimumab}

Belimumab is a B-lymphocyte stimulator-specific inhibitor that was first approved for treatment of SLE in 2011. It was the first monoclonal antibody approved to treat SLE. ${ }^{7}$ B-lymphocyte stimulator plays a critical role in B-cell survival; thus, its inhibition increases apoptosis of autoreactive B cells involved in the pathogenesis of SLE. More recently, belimumab was approved for pediatric SLE in April 2019 based on the PLUTO study, a phase 2 randomized, double-blind study of 93 patients. ${ }^{8}$ Although patients with cutaneous manifestations of lupus were included in trials for belimumab, they lacked CLE-specific outcome measurements to truly evaluate the efficacy in treating skin disease. ${ }^{9}$ This medication currently is not approved by the US Food and Drug Administration (FDA) for CLE; however, it is used off label in some cases for recalcitrant disease. ${ }^{10}$

\section{Baricitinib}

Baricitinib is a selective and reversible inhibitor of JAK1 and JAK2 that was granted fast-track status by the FDA in December 2018. In a phase 2 trial, baricitinib was superior to placebo plus standard of care, primarily for arthritis and lupus nephritis. ${ }^{11}$ Although improvement of cutaneous disease was measured as an end point, it did not show significant improvement in disease. The presence of skin disease was high, but the activity of disease was low, which can make it difficult to show meaningful improvement, as there is not much room for patients to objectively improve. ${ }^{12}$ Showing meaningful improvement in skin disease often is difficult in phase 2 trials, especially when the trial design is focused on SLE rather than CLE activity. Further studies of baricitinib that include more severe patients with CLE disease are needed to truly understand its effects on the skin.

\section{Lenalidomide}

There have been several CLE studies in the last several years surrounding lenalidomide, an analog of thalidomide. ${ }^{13-15}$ This molecule has a number of immunomodulatory effects including antiangiogenic effects, increased natural killer cell-dependent cytotoxicity, and cytokine and interleukin inhibition. Lenalidomide is of particular interest in treating CLE, as it was shown to be more potent than thalidomide at low doses and with a better side-effect profile. Multiple small, open-label trials have shown lenalidomide to be both safe and efficacious in the treatment of CLE. ${ }^{13,14}$ In addition, iberdomide, a derivative of lenalidomide, recently completed a phase 2 dose-escalation study showing improvement in both SLE and

From Corporal Michael J. Crescenz VA Medical Center, Philadelphia, Pennsylvania, and the Department of Dermatology, Perelman School of Medicine, University of Pennsylvania, Philadelphia.

Dr. Borucki reports no conflict of interest. Dr. Werth has received honoraria from Biogen, Celgene Corporation, Eli Lilly \& Company, GlaxoSmithKline, and Medlmmune and has received grants from Biogen and Celgene Corporation.

This project was supported by the Department of Veterans Affairs Veterans Health Administration, Office of Research and Development, Biomedical Laboratory Research and Development.

Correspondence: Victoria P. Werth, MD, Department of Dermatology, Perelman Center for Advanced Medicine, Ste 1-330A, 3400 Civic Center Blvd, Philadelphia, PA 19104 (werth@pennmedicine.upenn.edu). 
CLE end points. ${ }^{16} \mathrm{~A}$ phase $2 \mathrm{~b}$ proof-of-concept study currently is underway (ClinicalTrials.gov Identifier NCT03161483).

\section{Monoclonal Antibodies}

Many developing therapies target specific components of the type I interferon pathway, which is a primary driver of CLE lesions. Innate immune system pathways involving type I interferon were shown to be active in the pathogenesis of CLE, and levels of interferon correlate with skin disease activity. ${ }^{17}$ One molecule in development that targets this pathway is BIIB059, a humanized IgG1 monoclonal antibody that binds to blood dendritic cell antigen 2. This cell surface protein is uniquely expressed on plasmacytoid dendritic cells, which are the main source of type I interferon overproduction in SLE. The binding of this antibody to the blood dendritic cell antigen 2 receptor both blocks type I interferon production and decreases the overall number of active plasmacytoid dendritic cells present. ${ }^{18}$ In the completed phase $1 \mathrm{~b}$ study, a response in cutaneous disease was shown through a reduction in the CLE disease area and severity index score following single-dose administration. ${ }^{19}$ More recently, a phase 2 study met primary end points in both SLE and CLE compared to placebo. ${ }^{20}$

Anifrolumab is a human IgG1k monoclonal antibody that binds to type I interferon receptor, blocking all type I interferon signaling. Following a successful phase 2 trial, it failed to meet its primary end point in its first phase 3 trial. ${ }^{21}$ Several secondary end points suggested a clinical benefit. A second phase 3 trial of 362 patients randomized to treatment with anifrolumab or placebo over 48 weeks showed anifrolumab to be superior to placebo for multiple end points, including the overall disease primary end point as well as a notable reduction in skin activity. ${ }^{22}$

\section{Final Thoughts}

Outside of the approval of belimumab, there have been no new FDA-approved treatments for SLE since the approval of antimalarial agents nearly 50 years ago. For CLE specifically, there is an even greater scarcity of evidencebased treatments. Recently studied medications, such as belimumab and lenalidomide, are available off label for CLE patients when other options have failed. Recent studies have evaluated the efficacy of these agents in the treatment of CLE using the CLE disease area and severity index. ${ }^{10,13,14}$ Enrollment in CLE trials is difficult due to the rarity of the disease, and careful attention must be paid to evaluating skin end points. As experts in CLE and the nuances of these assessments, it is critical that dermatologists be involved in clinical trials. Future SLE trials must consider CLE as an important end point for CLE patients to get access to much-needed novel therapies.

\section{REFERENCES}

1. Bernatsky S, Boivin JF, Joseph L, et al. Mortality in systemic lupus erythematosus. Arthritis Rheum. 2006;54:2550-2557.

2. Vasquez R, Wang D, Tran QP, et al. A multicentre, cross-sectional study on quality of life in patients with cutaneous lupus erythematosus. $\mathrm{Br} J$ Dermatol. 2013;168:145-153.
3. Klein R, Moghadam-Kia S, Taylor L, et al. Quality of life in cutaneous lupus erythematosus. J Am Acad Dermatol. 2011;64:849-858.

4. Klein R, Moghadam-Kia S, LoMonico J, et al. Development of the CLASI as a tool to measure disease severity and responsiveness to therapy in cutaneous lupus erythematosus. Arch Dermatol. 2011; 147:203-208.

5. Jakhar D, Kaur I. Potential of chloroquine and hydroxychloroquine to treat COVID-19 causes fears of shortages among people with systemic lupus erythematosus. Nat Med. 2020;26:632.

6. American College of Rheumatology. Quinacrine shortage \& what the ACR is doing about it. https://www.the-rheumatologist.org/article /quinacrine-shortage-what-the-acr-is-doing-about-it/. Published February 8, 2019. Accessed May 15, 2020.

7. Dubey AK, Handu SS, Dubey S, et al. Belimumab: first targeted biological treatment for systemic lupus erythematosus. J Pharmacol Pharmacother. 2011;2:317-319.

8. Brunner H, Abud-Mendoza C, Viola D, et al. Efficacy and safety of intravenous belimumab in children with systemic lupus erythematosus [abstract]. Arthritis Rheumatol. 2018;70(suppl 10). https://acrabstracts.org labstract/efficacy-and-safety-of-intravenous-belimumab-in-children-with -systemic-lupus-erythematosus/. Accessed May 7, 2020.

9. Hui-Yuen JS, Reddy A, Taylor J, et al, Safety and efficacy of belimumab to treat systemic lupus erythematosus in academic clinical practices. J Rheumatol. 2015;42:2288-2295.

10. Vashisht P, Borghoff K, O'Dell JR, et al. Belimumab for the treatment of recalcitrant cutaneous lupus. Lupus. 2017;26:857-864.

11. Wallace DJ, Furie RA, Tanaka Y, et al. Baricitinib for systemic lupus erythematosus: a double-blind, randomised, placebo-controlled, phase 2 trial. Lancet. 2018;392:222-231.

12. Werth VP, Merrill JT. A double-blind, randomized, placebo-controlled, phase II trial of baricitinib for systemic lupus erythematosus: how to optimize lupus trials to examine effects on cutaneous lupus erythematosus. Br J Dermatol. 2019;180:964-965.

13. Cortés-Hernández J, Ávila G, Vilardell-Tarrés M, et al. Efficacy and safety of lenalidomide for refractory cutaneous lupus erythematosus. Arthritis Res Ther. 2012;14:R265.

14. Okon L, Rosenbach M, Krathen M, et al. Lenalidomide in treatmentrefractory cutaneous lupus erythematosus: efficacy and safety in a 52-week trial. J Am Acad Dermatol. 2014;70:583-584.

15. Fennira F, Chasset F, Soubrier M, et al. Lenalidomide for refractory chronic and subacute cutaneous lupus erythematosus: 16 patients. J Am Acad Dermatol. 2016;74:1248-1251.

16. Furie R, Werth V, Gaudy A, et al. A randomized, placebo-controlled, double-blind, ascending-dose, safety, and pharmacokinetics study of CC-220 in subjects with systemic LUPUS erythematosus [abstract]. Arthritis Rheumatol. 2017;69(suppl 10). https://acrabstracts.org /abstract/a-randomized-placebo-controlled-double-blind-ascending -dose-safety-and-pharmacokinetics-study-of-cc-220-in-subjects-with -systemic-lupus-erythematosus/. Accessed May 7, 2020.

17. Braunstein I, Klein R, Okawa J, et al. The interferon-regulated gene signature is elevated in subacute cutaneous lupus erythematosus and discoid lupus erythematosus and correlates with the cutaneous lupus area and severity index score. Br J Dermatol. 2012;166:971-975.

18. Kim JM, Park SH, Kim HY, et al. A plasmacytoid dendritic cellstype I interferon axis is critically implicated in the pathogenesis of systemic lupus erythematosus. Int J Mol Sci. 2015;16:14158-14170.

19. Furie R, Werth VP, Merola JF, et al. Monoclonal antibody targeting BDCA2 ameliorates skin lesions in systemic lupus erythematosus. J Clin Invest. 2019;129:1359-1371.

20. Werth V, Musselli C, Furie R, et al. BIIB059, a humanized monoclonal antibody targeting BDCA2 on plasmacytoid dendritic cells (pDC), shows dose-related efficacy in the phase 2 LILAC study in patients (pts) with active cutaneous lupus erythematosus (CLE). Ann Rheum Dis. In press.

21. Furie R, Morand EF, Bruce I, et al. Type I interferon inhibitor anifrolumab in active systemic lupus erythematosus (TULIP-1): a randomised, controlled, phase 3 trial. Lancet Rheumatol. 2019;1:E208-E219.

22. Morand EF, Furie R, Tanaka Y, et al. Trial of anifrolumab in active systemic lupus erythematosus. N Engl J Med. 2020;382:211-221. 